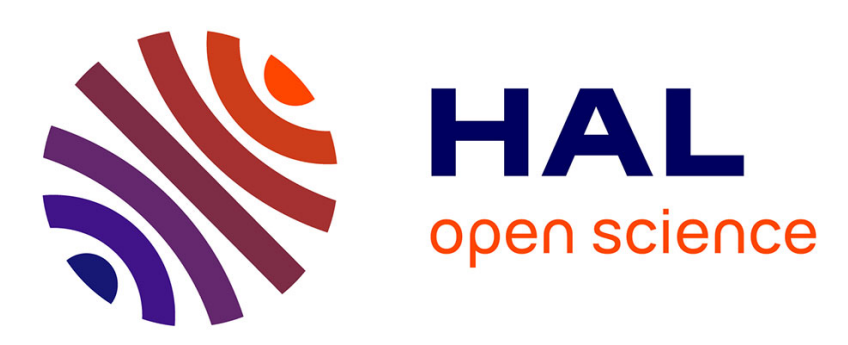

\title{
Hypothèse d'un dysfonctionnement émotionnel chez des adolescents présentant des comportements à risque: une étude exploratoire
}

Vincent Bréjard, Jean-Louis Pedinielli, Georges Rouan

\section{- To cite this version:}

Vincent Bréjard, Jean-Louis Pedinielli, Georges Rouan. Hypothèse d'un dysfonctionnement émotionnel chez des adolescents présentant des comportements à risque : une étude exploratoire. L'Encéphale, 2006, 32 (4 part 1), pp.413-420. 10.1016/S0013-7006(06)76182-6 . hal-01314709

HAL Id: hal-01314709

https://hal-amu.archives-ouvertes.fr/hal-01314709

Submitted on 12 Sep 2016

HAL is a multi-disciplinary open access archive for the deposit and dissemination of scientific research documents, whether they are published or not. The documents may come from teaching and research institutions in France or abroad, or from public or private research centers.
L'archive ouverte pluridisciplinaire HAL, est destinée au dépôt et à la diffusion de documents scientifiques de niveau recherche, publiés ou non, émanant des établissements d'enseignement et de recherche français ou étrangers, des laboratoires publics ou privés. 


\title{
Hypothèse d'un dysfonctionnement émotionnel chez des adolescents présentant des comportements à risque : une étude exploratoire
}

\author{
V. BREJARD (1), J.-L. PEDINIELLI (1), G. ROUAN (1)
}

\section{Emotional dysfunction hypothesis in adolescent with problem behaviour : an exploratory study}

Summary. Introduction. Problem behaviour in adolescents today has become a real public health problem, because of the potentially heavy consequences on their mental integrity. Although a number of studies have pointed out the importance of emotional functioning in this phenomenon, its characteristics have not been clearly defined. In the field of pathological emotional functioning, the aim of the study was to explore the differences in emotion variables, verbal emotion expression ability and behaviour, and notably behaviour inhibition ability. We hypothesised that we would observe significant differences between adolescents who exhibit problem behaviour and those who do not, in the three fields of subjective emotional experience, emotion verbalization, and behavioural reaction. Method. The sample for this investigation consisted in 40 high-school students - divided into two groups of 20 subjects: The first group : Problem Behaviour (PB), mean age : $15.2 \pm 1.2$, sex ratio (M/F) : 14/6, and the second group : No Problem Behaviour (NPB) mean age : I4.6 \pm 1.5 , sex ratio $(M / F): 13 / 6$. Subjects were included into the $P B$ group by completing a short questionnaire assessing problem behaviour (including verbal and behavioural violence, multiple exclusions of more than one day, substance use, and other risk-taking behaviour). To explore the emotional reactivity to stimuli, we provoked emotions using films excerpts with three situations: anger, joy and sadness. We assessed the subjective experience of emotion using the Differential Emotion Scale (DES, Izard, 1972), the verbal emotion expression by quantitative discourse analysis, and motor activity using the number of movements during stimuli exposure. Results. Statistical analysis (repeated ANOVAs) showed significant differences $(p<0.0001)$ in subjective emotional intensity, motor activity ( $p<0.0001)$, and verbai emotion expression ability $(p<0.0001)$ between the two groups. Adolescents with problem behaviour presented lower subjective emotional experience intensity, greater motor activity and reported less emotional words to describe their feelings. Discussion. Our results show an emo-tional dysfunction in adolescents with problem behaviour. These results are consistent with previous investigations, supporting the importance of emotional processing in the ability of people to cope with environment. This dysfunction may be linked to a pathology in the ability to "feel " the emotions. Implications for clinical practice are discussed.

Key words : Adolescent ; Dysfunctional processing ; Emotion ; Problem behaviour.

Résumé. Les comportements à risque chez les adolescents sont responsables d'un nombre croissant de situations met-tant en jeu leur intégrité physique et psychique. Il apparaît que de manière centrale, le fonctionnement émotionnel soit impliqué dans le fait que certains adolescents s'engagent dans ce type de comportements. Dans cette étude, nous avons tenté de montrer qu'ils sont la conséquence d'un dys-fonctionnement cognitivoémotionnel à situer au niveau de
L'intensité de l'éprouvé subjectif. Nos résultats ont été obte-nus après comparaison de deux groupes d'adolescents de 20 sujets chacun âgés de 14 à 16 ans : un groupe présentant des comportements à problème, un groupe n'en présentant pas. À partir d'une induction d'émotions par audiovisuel, nous avons évalué l'éprouvé subjectif par l'échelle d'émotions dif-férentielles (Izard). Nous avons ensuite évalué les capacités d'expression verbale des émotions par un entretien semi-

(1) Centre PsyCLE, EA 3273, UFR Psychologie, Sciences de l'Éducation, Université de Provence, 29, avenue Schuman, 13621 Aix-enProvence cedex 1.

Travail reçu le 24 janvier 2005 et accepté le 16 mai 2005.

Tirés à part : V. Brejard (à l'adresse ci-dessus). 
directif, et enfin la capacité à inhiber les réactions motrices par une grille d'observation. Nos résultats montrent après analyse de la variance que les adolescents présentant des comportements à risque ressentent les émotions de manière moins intense, verbalisent plus difficilement leurs éprouvés subjectifs et présentent un niveau supérieur d'activité motrice.Cesrésultats sont comparables à ceux obtenus dans d'autres travaux, et soulignent l'importance du fonctionne-ment émotionnel et de ses relations avec la sphère motrice dans la problématique comportementale à l'adolescence.

Mots clés : Adolescent ; Comportements à problèmes ; Émotion ; Processus dysfonctionnel.

\section{INTRODUCTION}

Les adolescents ont actuellement tendance à s'engager dans des comportements dont la caractéristique est de présenter un danger à plus ou moins long terme, pour eux-mêmes et pour autrui (9). Même si ces comportements restent le fait d'une minorité de jeunes, les conséquences potentielles n'en sont pas moins dramatiques, du fait de leur répétition. De plus, une part non négligeable des adolescents présente la particularité de "cumuler » les comportements problématiques $(5,19)$. Ces derniers incluent des conduites à risque (automobiles, sexuelles), des comportements de consommation et d'abus de substances (cannabis, alcool, tabac principalement), et des comportements agressifs. L'ensemble de ces comportements est actuellement regroupé sous la terminologie générique de " comportements à risque ", traduction littérale de l'expression anglo-saxonne risk-taking behaviours. Aux Etats-Unis, pour rendre compte de cette " comorbidité comportementale ", Jessor et al. (12) ont proposé le terme de «syndrome de comportements à problème ». Ceci marque bien la spécificité que constitue la présence chez ces adolescents non pas d'un seul type de comportement, mais de plusieurs. En outre, il semble que les adolescents présentant ce type de comportements aient les plus grandes difficultés à ne pas utiliser le registre comportemental lorsqu'ils sont confrontés à une situation qui leur pose problème ou qui prend une signification particulière pour eux. Ce qui pose question n'est pas qu'ils utilisent le comportement, mais que cette réaction devienne exclusive d'autres modalités de transaction entre l'individu et l'environnement. Actuellement, les recherches s'orientent de plus en plus sur la compréhension des rapports entre émotions et comportement, et plus précisément sur le rôle de la régulation des émotions comme facteur d'explication des comportements à risque .

\section{ÉMOTION ET COMPORTEMENT}

Différentes hypothèses ont été formulées pour tenter de rendre compte des relations potentielles entre fonctionnement émotionnel et comportement. Elles s'inscrivent principalement dans deux grands axes théoriques : l'un proposé dans une perspective psychanalytique, et l'autre dans une perspective cognitivo-émotionnelle.

\section{Modèles psychanalytiques}

Un certain nombre de travaux, principalement dans une optique psychanalytique et sur la thématique spécifique des addictions $(10,18)$ mettent l'accent sur la tendance à utiliser les sensations en lieu et place de l'émotion/affect (11). Les émotions seraient ainsi vécues comme trop intolérables pour le Moi et remettraient en cause le sentiment d'identité, voire les assises narcissiques. Une autre hypothèse porte sur la réactualisation de la problématique œdipienne avec un objet vécu comme trop proche, qui est alors attaqué* (23). L'objet suscite une trop grande excitation libidinale, et l'adolescent n'a d'autre solution que de tenter d'annuler ce sentiment de dépendance à l'objet soit en l'agressant pour tenter d'inscrire une séparation, soit en se réfugiant dans l'abus de substance, voire la dépendance, afin d'annuler l'emprise de l'objet externe et de sauvegarder un sentiment de contrôle et d'omnipotence préservant les assises narcissiques. D'autres auteurs principalement nord-américains (28) ont proposé pour caractériser les individus présentant des troubles psychopathologiques à expression comportementale et à forte composante émotionnelle, la notion de developmental arrest ou arrêt développemental. Selon cette théorie, la problématique ne serait pas à rechercher du côté du conflit œdipien et de ses avatars, mais d'un déficit structural à un niveau de développement pré-œdipien. Ceci aurait pour conséquence une vulnérabilité émotionnelle majeure face aux éléments de l'environnement et une tendance à passer à l'acte lorsque l'individu se trouve confronté à une situation vécue comme dépassant ses ressources psychiques. Cette notion d'arrêt du développement sera retrouvée plus loin dans une optique développementale.

\section{Modèles cognitivo-émotionnels}

D'autres travaux se référant à un paradigme cognitivoémotionnel ont également insisté sur l'importance du fonctionnement émotionnel et de ses troubles dans différents comportements à problème. Ils soulignent l'existence de phénomènes pathologiques dans le traitement de l'information émotionnelle chez les adolescents présentant des traits psychopathiques (17). Ainsi a été mis en évidence un lien, existant dès l'enfance, entre des troubles dans le développement de la conscience et de la capacité à ressentir des émotions, et une difficulté dans l'inhibition comportementale $(1,13)$. D'autres recherches ont démontré l'influence de facteurs de personnalité comme l'impulsivité, associée à l'expérience d'émotions négatives dans la prédisposition à s'engager dans des comportements déviants, ces éléments étant susceptibles d'amener le sujet à privilégier une réponse comportementale afin de réduire un état de tension, ou encore des états émotionnels tels que la peur, la tristesse ou encore l'anxiété (4).

Dans les travaux portant sur les conduites à risque, ou encore sur les addictions, la psychopathologie des émotions et particulièrement l'alexithymie est souvent mise en

\footnotetext{
* La répétition ayant valeur d'agression et de suppression de l'obje2
} 
avant comme facteur explicatif du comportement étudié $(2,16)$. Ce concept, proposé par Sifneos (26) (du préfixe privatif, mots, émotions) signifie littéralement " absence de mots pour décrire les émotions ». II recouvre un certain nombre de caractéristiques émotionnelles et cognitives chez des patients présentant des symptômes psychosomatiques : une difficulté majeure à identifier et à exprimer verbalement leurs émotions, une pauvreté de la vie fantasmatique et une tendance à l'agir (22). Un éclairage intéressant du phénomène alexithymique est apporté par la théorie des niveaux de conscience émotionnelle (15). Selon cette théorie, le développement émotionnel suivrait un parcours similaire à celui décrit par Piaget dans le cadre du développement cognitif. L'alexithymie serait alors une conséquence d'un arrêt à un stade infantile du développement émotionnel, équivalent au stade sensori-moteur. Le sujet aurait ainsi des difficultés importantes à reconnaître ses propres états subjectifs, mais également ceux d'autrui. Le vécu émotionnel serait essentiellement centré sur les perceptions des sensations internes avec une pauvreté dans la capacité à expérimenter des états subjectifs différenciés.

\section{Fonction des émotions}

Toujours dans une approche cognitivo-émotionnelle, un éclairage intéressant sur les rapports entre stimulus environnemental, émotion et comportement est proposé par la conception de Scherer (25) sur la fonction des émotions. Selon lui, le rôle fondamental de l'émotion consiste à instaurer un « temps de latence » entre l'environnement et la réponse comportementale. Ainsi, à la différence des approches purement psychophysiologiques qui postulent que l'émotion consiste en une réponse avant tout physiologique qui détermine un comportement motivé sur l'exemple " stimulus-réponse »- de type perception de l'ours $>$ fuite $>$ peur -, Scherer introduit la notion de fonction de découplage de l'émotion entre un événement et la réponse (comportementale ou non) que l'individu y fera. L'émotion permettrait donc d'instaurer une phase de traitement et d'analyse de l'environnement, afin de donner le temps à l'individu de réagir de la manière la plus adéquate possible. Ceci n'exclut bien évidemment pas la possibilité d'une réaction en urgence si un besoin ou un danger vital se faisait sentir. L'intérêt de cette conception est qu'elle propose un modèle de compréhension de l'émotion comme moyen de réguler les comportements de manière à ce qu'ils soient adaptés autant que possible à l'environnement. On peut ainsi envisager que la description de Scherer dans le fonctionnement normal puisse prendre un aspect pathologique lorsqu'un individu est dans l'incapacité d'utiliser ses émotions comme moyen de réguler ses comportements.

\section{OBJECTIFS}

Les objectifs de notre recherche sont:

- de montrer qu'il existe un dysfonctionnement émotionnel fondamental chez les adolescents présentant un syndrome de comportement à problème. De nombreux travaux ont en effet montré l'influence de variables émotionnelles dans différents comportements pathologiques. De plus, les variables émotionnelles entretiennent un lien important avec la capacité à réguler les comportements ;

- de comprendre les modalités de ce dysfonctionnement afin de proposer des aménagements au niveau des prises en charge.

\section{MÉTHODOLOGIE}

\section{Participants}

Les sujets ont été recrutés dans un collège de la région Provence, et ont répondu favorablement à une demande de participation à une recherche sur « ce qu'on ressent en regardant un film ». Ils étaient donc volontaires pour participer à cette étude. Les adolescents étaient en outre tous scolarisés dans le système « classique » (et pas dans les filières d'adaptation et d'intégration scolaire), ce qui minimise le risque de biais liés à des retards intellectuels ou dans l'acquisition du langage. Leur accord ainsi que celui des parents a été demandé. L'effectif total de l'échantillon était de 40 élèves. Ces adolescents ont été répartis en deux groupes :

- un groupe "CAP " (conduites à problème) $(n=20)$ (sujets présentant des comportements à problème), d'un âge moyen de $15,2 \pm 1,2$, avec un sex- ratio $(H / F)$ de $14 / 6$;

- un groupe "NCAP » $(n=20)$ : sujets ne présentant pas de comportements particuliers, dont l'âge moyen est $14,6 \pm 1,5$ an, avec un sex-ratio $(H / F)$ de 13/6.

Les élèves du groupe CAP ont été soumis à un questionnaire d'évaluation comportementale; nous avons construit ce dernier à partir d'entretiens avec les enseignants et d'observations effectuées en collège, comportant les critères suivants : présence de comportements antisociaux (violence comportementale et verbale à l'égard des pairs, des enseignants, des surveillants), exclusions multiples dépassant le cadre d'une journée, consommation de substances psycho-actives (tabac, alcool, cannabis), échec scolaire, autres comportements associés (rester tard le soir hors du domicile parental, conduites à risque : jeu du foulard, conduite scooter...). Ces critères ont fait l'objet d'une triple évaluation par les pairs, un enseignant, un surveillant. Chacun des sujets inclus dans notre recherche présentait au moins trois de ces critères.

\section{Hypothèses}

1) Les adolescents ayant des comportements à problème (CAP) ressentent les émotions de manière moins intense que les adolescents n'en présentant pas (NCAP).

2) Les adolescents CAP verbalisent un nombre de mots à contenu émotionnel moins important que les adolescents NCAP.

3) Les adolescents CAP ont un niveau d'activation motrice supérieur aux adolescents NCAP : ils présentent plus d'agitation motrice. 


\section{Outils}

\section{Induction d'émotions}

Nous avons choisi d'utiliser un protocole d'induction d'états subjectifs émotionnels par audiovisuel à l'aide d'extraits de films en nous inspirant de travaux effectués auprès d'adultes $(8,24)$. Notre protocole comportait l'induction de trois types d'émotions : colère, joie et tristesse. L'induction était effectuée avec des extraits de films dont les contenus ont été évalués dans le cadre d'un prétest auprès d'un échantillon de 69 adolescents, afin de vérifier qu'ils induisaient bien respectivement les émotions à évaluer. Ils ont été présentés aux sujets de l'étude sur un écran d'ordinateur portable. Les émotions ont été évaluées avec l'échelle d'émotions différentielles (DES) d'Izard (21). Cette étude préliminaire a permis d'évaluer la capacité des extraits de film à induire des états subjectifs différenciés. La description des séquences est présentée dans le tableau $I$.

TABLEAU I. - Extraits de films utilisés pour l'induction d'émotions.

\begin{tabular}{lll}
\hline $\begin{array}{l}\text { Stimulation } \\
\text { choquante }\end{array}$ & $\begin{array}{l}\text { Extrait du film } \\
\text { "La ligne }\end{array}$ & $\begin{array}{l}\text { Un homme est emprisonné et } \\
\text { condamné à mort. Sa seule amie }\end{array}$ \\
$\begin{array}{l}\text { (dégoût, } \\
\text { révolte) }\end{array}$ & verte " & $\begin{array}{l}\text { est une souris. Un gardien la tue } \\
\text { brutalement en l'écrasant d'un } \\
\text { coup de talon. L'homme crie et } \\
\text { pleure }\end{array}$
\end{tabular}

Stimulation Extrait du film Un homme est amoureux d'une positive "Mary à tout très jolie fille. Il lui rend visite. Elle (joie) prix » le laisse seul avec son petit chien. Celui-ci grogne, puis se jette sur l'entrejambe du héros, qui se bat avec lui, puis finit par le jeter par la fenêtre

Stimulation Extrait du film Un homme et une femme se triste "Rencontre rencontrent dans un café. Ils se (tristesse) avec Joe séduisent puis chacun s'en va de Black » son côté. Au moment où elle se retourne pour le regarder une dernière fois, il se fait renverser par une auto

\section{Échelle d'émotions différentielles}

Après chaque extrait de film, l'éprouvé subjectif émotionnel a été mesuré par l'échelle DES d'lzard. Cette échelle laisse peu de place à la désirabilité sociale, car les sujets doivent répondre à un seul mot par item sur une échelle en 5 points. Ceci entraîne moins facilement une tendance au conformisme, du moins pour des adolescents. Cette échelle compte 9 facteurs, contenant de 1 à 7 items : sensitivité ( 7 items), joie ( 3 items) qui peut être interprétée comme la sensibilité aux émotions négatives, colère (3 items), paranoïa (4 items), surprise ( 3 items), attention ( 3 items), découragement ( 3 items), alerte (1 item), dégoût ( 3 items). Le sujet doit coter sur une échelle en 5 points l'intensité de son éprouvé, de 0 , « jamais », à 4 « très fortement ».

\section{Niveau d'activité motrice}

Afin d'évaluer le niveau d'activation comportementale, nous avons utilisé les vidéos filmées pendant l'exposition aux extraits de films. Le protocole des observations a été analysé avec une grille d'observation de l'expression comportementale, qui comportait trois dimensions : les mouvements effectués par les membres (gestes des bras et des jambes), les modifications posturales (se penche en avant, se recule), les déplacements (se lever de sa chaise, se rasseoir). Nous avons pu ainsi quantifier dans chaque secteur le nombre de manifestations qualitatives produites par chaque sujet, de manière à obtenir un score global, pouvant représenter le niveau d'activation motrice de chaque sujet, et ainsi obtenir un indicateur de son aptitude à inhiber la sphère motrice.

\section{Capacité à verbaliser les émotions}

La capacité de verbalisation des éprouvés subjectifs ressentis pendant le visionnage des extraits de film a été évaluée grâce à un entretien semi-directif (7). Cette technique permettait d'offrir un support d'expression verbale aux sujets ; elle offrait de surcroît l'avantage de s'assurer de l'absence de conséquences négatives de ce cadre expérimental.

Nos questions se limitaient à deux phrases:

- «Peux-tu me dire ce que tu as ressenti pendant les films?»

- "Qu'est-ce que tu en as pensé ? "

Ces questions volontairement ouvertes permettaient la libre expression des sujets sur leur ressenti, tout autant que de critiquer ou d'exprimer une réaction possible. Les entretiens ont fait l'objet d'un enregistrement audio et ont ainsi pu être traités par une analyse de contenu de type quantitatif avec recherche d'indicateurs langagiers pertinents (7).

Nous avons retenu comme indicateurs de la capacité à verbaliser des émotions les adjectifs ayant trait aux émotions (drôle, triste, en colère, révolté, marrant, galère...) et les formes langagières décrivant le ressenti, l'éprouvé (verbes statifs : j'ai ressenti ; j'ai senti ; j'étais - plus élément émotionnel ; j'ai éprouvé...).

\section{Procédure}

Les sujets étaient reçus dans une pièce neutre, et étaient installés devant un ordinateur. II leur était alors demandé de se relaxer légèrement en se concentrant sur leur respiration et en ralentissant et approfondissant celleci, afin d'éviter au maximum l'effet d'états émotionnels « parasites » pouvant avoir été suscités par des événements juste avant l'induction d'émotion. Puis un extrait de film leur était proposé. Durant ce film, ils étaient euxmêmes filmés par une caméra vidéo. Après chaque extrait, les sujets devaient remplir le questionnaire, puis répondre à deux questions que nous développons plus loin. 
Les traitements statistiques (ANOVA, mesures répétées) ont été effectués avec le logiciel Statview 5.0® (CSAS Institute, 1999).

\section{RÉSULTATS}

Les résultas obtenus seront présentés en trois parties. En premier lieu, nous décrirons les réactions émotionnelles à l'induction d'émotions. Puis nous examinerons les manifestations comportementales pendant l'induction d'émotions. Enfin nous présenterons les résultats obtenus aux entretiens

\section{Réponse émotionnelle subjective en fonction du groupe, du type d'émotion et des émotions mesurées}

Afin d'évaluer dans quelle mesure les deux groupes diffèrent sur le plan de l'intensité émotionnelle, nous avons effectué une ANOVA $2 \times 3 \times 9$ sur leurs évaluations subjectives avec comme facteur expérimental inter-sujet le type de groupe (CAP/NCAP), et comme facteur intra-sujet le type de film (induction négative, positive, colère), et les sous-échelles de la DES. Les effets principaux des émotions de la DES avec un
$F(8,304)=119,86, p>0,0001$, ainsi que du type de film $[F(2,76)=12,21, p>0,0001]$, associés à une interaction entre ces facteurs $[F(16,608)=20,48, p>0,0001]$ montrent que les extraits de films induisent bien des états subjectifs différenciés.

En ce qui concerne le niveau de l'intensité émotionnelle ressentie, les adolescents présentant des troubles du comportement diffèrent significativement des adolescents du groupe contrôle, et ressentent les émotions de manière moins intense avec un $F(1,38)=24,523$, $\mathrm{p}<0,0001$.

Cependant, il existe des particularités notables en fonction du type d'induction, comme le montre le tableau II.

En effet, on remarque que si, d'une manière générale, les adolescents du groupe expérimental ressentent les émotions de manière moins intense que ceux du groupe contrôle, il existe certains résultats " paradoxaux ", tels que le fait d'éprouver du dégoût au lieu d'une émotion positive (joie) et de la joie au lieu d'une émotion négative (colère). On constate donc que chez les adolescents présentant des comportements à problème, non seulement les émotions induites ne sont pas systématiquement les mêmes que celles des adolescents du groupe contrôle, mais lorsqu'elles sont similaires, elles sont ressenties de manière moins intense.

TABLEAU II. — Récapitulatif des résultats à la DES : moyennes (écarts types), et différences intergroupes après analyse de la variance, plan à mesures répétées.

\begin{tabular}{|c|c|c|c|c|c|c|c|}
\hline Échelles de la DES & & Colère & & Joie & & Tristesse & \\
\hline & Type Gr & Moyenne & $\mathrm{p}$ & Moyenne & $\mathrm{p}$ & Moyenne & $\mathrm{p}$ \\
\hline \multirow{2}{*}{ Sensitivité } & 1 & $13,65(2,73)$ & 0,00 & $9,9(2,26)$ & 0,30 & $15,8(3,18)$ & 0,00 \\
\hline & 2 & $7(1,81)$ & & $9(2,06)$ & & $9,75(2,44)$ & \\
\hline \multirow[t]{2}{*}{ Joie } & 1 & $3,55(1,16)$ & 0,00 & $11,85(2,91)$ & 0,00 & $3,15(0,79)$ & 0,00 \\
\hline & 2 & $6,35(1,98)$ & & $6,15(2,18)$ & & $5,35(1,32)$ & \\
\hline \multirow[t]{2}{*}{ Colère } & 1 & $9,3(2,54)$ & 0,00 & $3,2(1,31)$ & 0,11 & $6,75(2,36)$ & 0,00 \\
\hline & 2 & $4,1(1,18)$ & & $4(1,54)$ & & $5,15(1,49)$ & \\
\hline \multirow[t]{2}{*}{ Paranoïa } & 1 & $7,5(2,29)$ & 0,00 & $5,2(0,97)$ & 0,89 & $8,8(1,86)$ & 0,11 \\
\hline & 2 & $4,95(1,09)$ & & $5,1(1,16)$ & & $6,9(1,89)$ & \\
\hline \multirow[t]{2}{*}{ Surprise } & 1 & $8,25(2,15)$ & 0,01 & $6,65(2,07)$ & 0,48 & $7,5(2,19)$ & 0,02 \\
\hline & 2 & $5,2(1,46)$ & & $5,95(2,17)$ & & $5,5(1,28)$ & \\
\hline \multirow[t]{2}{*}{ Attention } & 1 & $7,05(2,23)$ & 0,11 & $7,25(2,34)$ & 0,15 & $7(2,41)$ & 0,09 \\
\hline & 2 & $5,95(2,06)$ & & $5,9(2,19)$ & & $5,65(2,24)$ & \\
\hline \multirow[t]{2}{*}{ Découragement } & 1 & $7,35(2,85)$ & 0,00 & $3,2(1,12)$ & 0,34 & $10,3(2,59)$ & 0,00 \\
\hline & 2 & $4,45(1,14)$ & & $3,5(0,99)$ & & $6,25(1,54)$ & \\
\hline \multirow[t]{2}{*}{ Alerte } & 1 & $2,25(0,62)$ & 0,69 & $1,7(0,34)$ & 0,02 & $1,9(0,59)$ & 0,46 \\
\hline & 2 & $2,05(0,73)$ & & $1,15(0,12)$ & & $1,65(0,43)$ & \\
\hline \multirow{2}{*}{ Dégoût } & 1 & $10,45(3,12)$ & 0,00 & $5,3(1,36)$ & 0,01 & $12,35(3,54)$ & 0,00 \\
\hline & 2 & $6,8(2,27)$ & & $7,6(1,88)$ & & $6,65(1,69)$ & \\
\hline
\end{tabular}
Intensité moyenne des émotions rapportées à la DES après chaque extrait de film, pour chaque groupe [Gr 1 : adolescent du groupe sans com-
portements à risque (NCAR), Gr 2 : adolescents présentant des comportements à risque (CAR)]. 


\section{Niveau d'activité comportementale}

Une ANOVA similaire à celle effectuée sur les échelles de la DES a été faite pour l'évaluation de la capacité à inhiber les comportements. Les résultats obtenus diffèrent sensiblement de ceux obtenus avec la DES. On constate un effet significatif du type de groupe sur le nombre de manifestations motrices observées $F(1,38)=21,537$, $p<0,0001$. Le type de film n'induit pas de différence significative, et il n'y pas d'interaction entre le type de film et le type de groupe. Les adolescents présentant des troubles du comportement présentent plus de manifestations motrices que les adolescents du groupe témoin, et semblent donc avoir un niveau d'activation comportementale plus élevé que ces derniers face à une stimulation émotionnelle de l'environnement, comme le montre le tableau III.

TABLEAU III. - Récapitulatif des résultats (statistiques descriptives) à l'analyse de l'activité motrice, et différences intergroupes en fonction du type de stimuli (test : Analyse de le variance)

\begin{tabular}{lccc}
\hline & $\begin{array}{c}\text { Type } \\
\text { de groupe }\end{array}$ & Moyenne & $\begin{array}{c}\text { Niveau de } \\
\text { significativité }\end{array}$ \\
\hline Colère & 1 & $10,85(2,26)$ & $\boldsymbol{p}<\mathbf{0 , 0 0 8}$ \\
Joie & 2 & $13,85(3,87)$ & \\
& 1 & $11,7(2,07)$ & $\boldsymbol{p}<\mathbf{0 , 0 0 2}$ \\
Tristesse & 2 & $15,6(4,12)$ & \\
& 1 & $10,45(2,21)$ & $\boldsymbol{p}<\mathbf{0 , 0 0 7}$ \\
& 2 & $13,8(3,77)$ & \\
\hline
\end{tabular}

Gr 1 : adolescent du groupe sans comportements à risque (NCAR); Gr 2 : adolescents présentant des comportements à risque (CAR).

\section{Capacités de verbalisation des éprouvés subjectifs}

Nous avons effectué une ANOVA $2 \times 3$, avec comme variable indépendante le type de groupe (CAP/NCAP) et le type de stimulation (colère, joie, tristesse), et comme variable dépendante le nombre d'indicateurs langagiers à contenu émotionnel. Ceci nous a permis d'évaluer l'effet du type de film et du type de groupe sur les capacités de verbalisation des éprouvés subjectifs. Les résultats montrent une absence d'effet du type de film $F(2,76)=0,621$, $p=0,54$. En revanche, il existe un effet principal du type de groupe $F(1,38)=70,111, p<0,0001$. On observe donc que les adolescents présentant des troubles du comportement utilisent significativement moins de mots et d'expressions permettant de verbaliser les éprouvés subjectifs que les adolescents du groupe contrôle, et ce, quel que soit le type de film et le type d'émotion induite (tableau IV).

\section{DISCUSSION}

Dans cette étude, nous souhaitions mettre en évidence un mode de fonctionnement émotionnel spécifique des
TABLEAU IV. - Récapitulatif des résultats (statistiques descriptives) aux indicateurs verbaux émotionnels, et différences intergroupes en fonction du type de stimuli (test . Analyse de la variance).

\begin{tabular}{lccc}
\hline & $\begin{array}{c}\text { Type } \\
\text { de groupe }\end{array}$ & Moyenne & $\begin{array}{c}\text { Niveau de } \\
\text { significativité }\end{array}$ \\
\hline Colère & 1 & $1,9(0,98)$ & $\boldsymbol{p}<\mathbf{0 , 0 0 1}$ \\
Joie & 2 & $0,7(0,21)$ & \\
& 1 & $1,85(0,84)$ & $\boldsymbol{p}<\mathbf{0 , 0 3}$ \\
Tristesse & 2 & $1,15(0,46)$ & \\
& 1 & $2,25(1,01)$ & $\boldsymbol{p}<\mathbf{0 , 0 0 1}$ \\
& 2 & $0,45(0,35)$ & \\
\hline
\end{tabular}

Gr 1 : adolescent du groupe sans comportements à risque (NCAR) ; $\mathrm{Gr} 2$ : adolescents présentant des comportements à risque (CAR).

adolescents présentant des comportements à risque. Les résultats obtenus valident nos hypothèses. Les adolescents présentant des comportements à risque semblent ressentir non seulement les émotions de manière moins intense sur le plan quantitatif, mais également sur le plan qualitatif avoir un éprouvé différent (type d'émotion ressentie). Par ailleurs, ils verbalisent significativement moins de mots exprimant un éprouvé subjectif. Enfin, ils présentent un niveau d'activation motrice plus élevé lorsqu'ils sont confrontés à un événement induisant un état émotionnel. Cela corrobore l'observation que l'on peut effectuer au niveau clinique.

Ces résultats doivent toutefois être analysés avec précaution du fait de limites inhérentes à cette étude. La première d'entre elles concerne l'absence d'évaluation standardisée des compétences des sujets du groupe expérimental dans le domaine de l'expression verbale. En effet, il est possible qu'une partie de la difficulté rencontrée par ces adolescents dans l'expression de leurs éprouvés subjectifs soit liée à des compétences plus faibles dans les capacités de verbalisation en général du fait d'un moindre niveau culturel, d'un retard de langage, ou d'un moindre niveau intellectuel, qui tous peuvent être associés aux troubles des conduites. La seconde est liée à l'absence d'évaluation du niveau socio-culturel des sujets. Cette variable peut en effet influencer la verbalisation des éprouvés subjectifs, mais ceci renvoie potentiellement selon nous à un environnement dans lequel l'expression verbale de l'éprouvé subjectif (le partage social de l'émotion) n'est pas encouragée. Une des conséquences possibles est que le sujet n'est alors pas dans une situation où il est amené à mettre en place des stratégies de mentalisation de ses émotions afin de pouvoir les communiquer.

Cependant, si l'on regarde les résultats dans le détail, on constate que non seulement les adolescents CAP verbalisent moins d'éléments émotionnels, mais qu'en outre ils disent ressentir les émotions de manière moins intense. II est possible que les adolescents éprouvent des émotions fortes pendant la confrontation aux stimuli, mais décrivent une faible intensité ressentie, soit volontaireø 
ment (falsification du fait d'un biais de désirabilité sociale ou de désirabilité personnelle, les adolescents délinquants pouvant considérer les émotions moins acceptables que les adolescents non délinquants et pouvant donner des réponses plus conformes à l'image qu'ils se font d'eux-mêmes), soit involontairement (déni des émotions).

Au-delà de ces différents éléments, ces résultats pourraient également être expliqués par un fonctionnement mental de type pensée opératoire, caractérisé par la difficulté à reconnaître et verbaliser ses propres états subjectifs. De plus on sait que c'est précisément un type de fonctionnement analogue qui est retrouvé dans différents comportements, addictifs notamment $(16,20)$. Ceci amène à rediscuter cette hypothèse pour envisager le fait que ce fonctionnement puisse s'inscrire plutôt dans le cadre d'un déficit acquis dans la capacité à ressentir les émotions. En d'autres termes, les adolescents CAP pourraient ressentir les émotions de manière différente des autres adolescents et auraient des difficultés à les exprimer verbalement du fait d'une variation principalement quantitative dans l'intensité de l'éprouvé. Ainsi, il s'agirait non pas d'une tendance à recourir à l'action comme mode de régulation émotionnelle (2), ou d'une stratégie adaptative de type coping répressif (29), mais bien plutôt d'un phénomène d' " atténuation émotionnelle ».

Ce phénomène constituerait selon nous un mécanisme de défense destiné à réprimer les émotions avant qu'elles ne deviennent conscientes. II permettrait à l'adolescent de ne pas être confronté à des éprouvés subjectifs potentiellement déstructurants. Par ce phénomène adaptatif, il se produirait une réduction de l'intensité émotionnelle dont la conséquence observable serait l'incapacité à exprimer l'émotion par le biais du langage. Ceci pourrait s'expliquer par le fait que les émotions sont vécues comme des événements psychiques dangereux pour le sujet. Elles seraient alors susceptibles de porter atteinte à une homéostasie psychique que le mécanisme d'atténuation émotionnelle permet de maintenir dans une fonction similaire à celle de l'alexithymie $(15,20)$.

Nous pouvons, plus avant, comprendre ce phénomène comme une véritable « stratégie antipensée et antireprésentation » $(3,14)$, grâce à laquelle le sujet se protège au prix d'une incapacité à envisager une résolution de problème alternative au " comportemental ». Ainsi, les adolescents présentant des comportements à problème auraient une tendance à recourir à l'action du fait même qu'ils ont des difficultés à mentaliser, à " penser " les émotions, à leur donner une représentation psychique. L'une de leur caractéristique psychopathologique majeure serait ainsi une incapacité d'accès à un niveau fonctionnel suffisant de mentalisation des éprouvés subjectifs.

La principale conséquence de ce mécanisme de défense (au-delà de son effet sur l'éprouvé subjectif), si l'on rapproche ces résultats des propositions de Scherer, serait d'entraîner une pathologie fonctionnelle : l'atteinte de la fonction de découplage de l'émotion. Les adolescents ressentant les émotions moins intensément, cellesci ne peuvent alors jouer leur rôle de régulation des comportements, du fait d'une disparition de la phase intermé- diaire de "conscientisation " de l'émotion permettant l'orientation du comportement. L'activation comportementale et la tendance à l'action face à un stimulus restent donc privilégiées. Ceci se retrouve également dans nos résultats : les adolescents présentant des comportements à problème ont tendance à montrer plus d'agitation motrice lorsqu'ils sont confrontés à des stimuli émotionnels. Ce trouble de la perception interne des émotions, associé à cette perturbation de leur fonction régulatrice, pourrait à notre sens trouver son origine dans des événements du développement de ces adolescents, dont on connaît les trajectoires sociales et familiales le plus souvent dramatiques, faites de traumatismes et de situations d'instabilité. Ces caractéristiques environnementales pourraient fournir une explication possible du recours aux modalités pathologiques de fonctionnement émotionnel de ces adolescents, du fait d'un arrêt développemental à un stade où les sensations de tensions internes peuvent être réprimées avant de devenir des émotions.

\section{CONCLUSION}

Une hypothèse séduisante permettant d'expliquer les causes de cet arrêt développemental peut être recherchée dans certains travaux psychanalytiques (post-kleiniens et post-hartmaniens pour l'essentiel) (28). En accord avec ces auteurs, nous pensons que cette vulnérabilité interne face aux contenus émotionnels pourrait trouver son origine dans des altérations précoces, traumatogènes, de la relation de l'enfant à son environnement. Des événements négatifs précoces associés à un milieu familial peu étayant auraient ainsi pu provoquer des arrêts développementaux ne donnant pas à l'enfant les équipements psychiques susceptibles de lui permettre de supporter divers contenus psychiques sans provoquer une menace pour son Self. Ainsi l'enfant puis l'adolescent, se trouvant dans l'incapacité de se constituer un sentiment de cohérence interne suffisant, ne peut que se réfugier dans le clivage entre sa propre subjectivité et ses états de tension interne. Cela a comme conséquence majeure l'incapacité à penser les émotions, et ainsi à les utiliser dans leur fonction régulatrice sans se sentir menacé dans son intégrité subjective. Cette double référence, voulue et soutenue par nous, à des axes épistémologiques distincts, nous paraît justifiée par les caractéristiques liées au phénomène étudié. Notre éclectisme théorique (psychanalytique, cognitivo-émotionnel) est une stratégie pour maintenir les pistes heuristiques ouvertes, stratégie de chercheur lorsque le phénomène est encore trop peu connu et en débats d'alternatives $(6,27)$. Mais cet éclectisme n'est pas seulement théorique, car nous sommes conscients des implications cliniques qu'il sous-tend. II amène à rediscuter les techniques psychothérapeutique susceptibles d'être utilisées dans la prise en charge de ces adolescents, en tenant compte de leurs difficultés d'élaboration et de confrontation aux éléments émotionnels. Ces résultats, s'ils s'avéraient confortés, nous paraissent avoir des implications non négligeables sur le plan des prises en charge thérapeutiques de ces patients. 


\section{Références}

1. BLAIR RJR. Responsivness to distress cues in the child with psychopathic tendencies. Pers Ind Differ 1999 ; 27 : 135-45.

2. BONNET A, PEDINIELLI JL, ROUAN G. Bien-être subjectif et régulation émotionnelle dans les conduites à risques : le cas de la plongée sous-marine. Encéphale 2003 ; 29 (6) : 488-95.

3. CATTEEUW M. La phobie du penser : étude des fonctionnalités des conduites addictives (thèse non publiée). Toulouse : Université du Mirail, 1999

4. CASPI A, BEGG D, DICKSON $\mathrm{N}$ et al. Personality traits predict health-risk behaviours : evidence from a longitudinal study. J Pers Soc Psychol 1997 ; 73 : 1052-63.

5. CHOQUET M, MARECHAL C. Alcoolisation des jeunes, consommation de drogues et troubles des conduites : approche épidémiologique et préventive. Alcoologie $1990 ; n^{\circ}$ sp : 32-7.

6. FEE D. Pathology and the Postmodern : Mental illness as Dicourse and Experience. Thousand Oaks, CAL : Sage, 2000.

7. GHIGLIONE R, BLANCHET A. Analyse de contenu et contenu d'analyse. Paris : Dunod, 1991.

8. GROSS JJ, LEVENSON RW. Emotion elicitation using films. Cog Emotion $1995 ; 9: 87-108$.

9. GUILBERT P, GAUTIER A, BAUDIER $F$ et al. Baromètre santé 2000 : les comportements des 12-25 ans, vol. 3.1 : synthèse des résultats nationaux et régionaux. Saint-Denis : INPES, 2004.

10. JEAMMET P. Addiction-dépendance-adolescence, réflexions sur leurs liens, conséquences sur nos attitudes thérapeutiques. In . Venisse JL, ed. Les nouvelles addictions. Paris : Masson, 1991 10-29.

11. JEAMMET $P$. Dépendance et séparation à l'adolescence, point de vue psychodynamique, In : Bailly $\mathrm{D}$, Venisse J, eds. Dépendance et conduites de dépendance. Paris: Masson, 1994 : 134-44.

12. JESSOR R, DONOVAN JE, COSTA F. Beyond adolescence : problem behavior and youg adult development. New York : Cambridge University Press, 1991.

13. KOCHANSKA G. Toward a synthesis of parental socialization and child temperament in early development of conscience. Child Dev $1993 ; 64: 325-47$.
14. LANE RD, SECHREST L, RIEDEL R. Pervasive emotion recognition deficit common to alexithymia and the repressive coping style. Psychosom Med $2000 ; 62: 492-501$.

15. LANE RD, SCHWARTZ GE. Levels of emotionnal awarness : a cognitive developmental theory and its application to psychopathology. Am J Psychiatry 1987 ; 144 : 133-43.

16. LOAS G, OTMANI O, LECERCLE C et al.. Relationships between the emotional and cognititve components of alexithymia and dependency in alcoholics. Psychiatr Res 2000 ; $96: 63-74$.

17. LONEY BR, FRICK PJ, CLEMENTS CB et al. Callous-unemotional traits, impulsivity, and emotional processing in adolescents with antisocial behavior problems. J Clin Child Adolesc Psychol 2003 ; 32 : 1-93.

18. MARCELLI D., BRACONNIER A. Adolescence et psychopathologie, $5^{\mathrm{e}}$ éd. Paris : coll Les âges de la vie, Masson, 2000.

19. MICHEL G. La prise de risque àl'adolescence. Paris : Masson, 2000.

20. MONTREUIL M, JOUVENT M, CARTON S et al. Parrallel visual information processing test. Psychother Psychosom $1991 ; 56: 212-9$.

21. OUSS L, CARTON S, JOUVENT R et al. Traduction et validation de l'échelle d'émotions différentielles d'Izard. Exploration de la qualification verbale des émotions. Encéphale 1990 ; 16 : 453-8.

22. PEDINIELLI JL. Psychosomatique et alexithymie. Paris : PUF, 1992.

23. PEDINIELLI JL, ROUAN G, GIMENEZ G et al. Psychopathologie des conduites à risque. Ann Med Psychol $2005 ; 163$ : 30-6.

24. PHILIPPOT P. Inducing and assessing differentiated emotion-feeling states in the laboratory. Cogn Emotion $1993 ; 7: 171-93$.

25. SCHERER KR. Emotion serves to decouple stimulus and response. In : Ekman P, Davidson RJ, eds. The nature of emotion : fundamental questions. New York/Oxford : Oxford University Press, 1994 : 127-30.

26. SIFNEOS PO. The prevalence of « alexithymia » characteristics in psychosomatic patients. Topics of psychosomatic research. Bâle : Karger, 1972.

27. SMITH JA, HARRE R, VAN LANGENHOVE LV. Rethinking Methods in Psychology. London : Sage, 1995.

28. STOLOROW RD, LACHMANN FM. Psychoanalysis of developmental arrests, $6^{\mathrm{e}}$ ed. Madison, Conn : International University Press, 1989.

29. WEINBERGER DA. The construct validity of repressive coping style. In : Singer JL, ed. Repression and Dissociation. Chicago : University of Chicago Press, 1990 : 337-86. 